Article

\title{
Efficient synthesis of pyrano[2,3-d]pyrimidinone and pyrido[2,3- $d$ ]pyrimidine derivatives in presence of novel basic ionic liquid catalyst
}

\author{
Omid Goli Jolodar, Farhad Shirini *, Mohadeseh Seddighi \\ Department of Chemistry, Faculty of Sciences, University of Guilan, Rasht 41335, Iran
}

\section{A R T I C L E I N F O}

\section{Article history:}

Received 21 January 2017

Accepted 15 March 2017

Published 5 July 2017

\section{Keywords:}

Basic catalyst

Multicomponent reaction

Pyrimidine derivative

Grinding

\begin{abstract}
A B S T R A C T
A basic ionic liquid, namely 1,1'-(butane-1,4-diyl)bis(1,4-diazabicyclo [2.2.2]octan-1-ium) hydroxide, was prepared and characterized using Fourier-transform infrared spectroscopy, ${ }^{1} \mathrm{H}$ nuclear magnetic resonance spectroscopy, and $\mathrm{pH}$ measurements. The ionic liquid was used for efficient promotion of the synthesis of pyrano[2,3- $d]$ pyrimidinone and pyrido[2,3- $d]$ pyrimidine derivatives at room temperature under grinding conditions. A simple procedure, short reaction time, high yields, non-column chromatographic separation, commercial availability of the starting materials, and recyclability of the catalyst are attractive features of this process.
\end{abstract}

(C) 2017, Dalian Institute of Chemical Physics, Chinese Academy of Sciences. Published by Elsevier B.V. All rights reserved.

\section{Introduction}

Highly efficient, selective, and cost-effective catalytic systems are the foundation of synthetic chemistry. Ionic liquids (ILs) have emerged as eco-friendly catalysts or catalysts/solvents in various fields of chemistry because of their distinctive properties such as high thermal stability, negligible vapor pressure, high loading capacity, and easy recycling [1].

Basic ILs have attracted great interest because, compared with inorganic bases such as $\mathrm{KOH}$ and $\mathrm{NaOH}$, they have good catalytic efficiencies, are non-corrosive, recyclable, and produce no waste [2]. This type of IL has been successfully used in the industrial production of drugs, fragrances, and chemical intermediates, and to accelerate various reactions such as Michael additions, Heck reactions, and Markovnikov additions [3,4].

Pyrano[2,3- $d$ ] pyrimidinones (A) and pyrido[2,3- $d$ ] pyrimidines (B) (Fig. 1) are two classes of nitrogen-containing heterocyclic compounds that show considerable pharmaceutical and biological activities, including anticancer, antitumor, antima- larial, antibacterial, antihypertensive, anti-inflammatory, hepatoprotective, cardiotonic, vasodilator, bronchiodilator, antifolate, and antiallergic activities [5-17]. They are also used in the preparation of dyes and pigments [18] and flavoring agents [19], and in luminescence chemistry [20].

The most simple and straightforward protocols for the preparation of pyrano[2,3-d]pyrimidinones and pyrido[2,3- $d]$ pyrimidines are based on three-component reactions of substituted aldehydes, malononitrile, and barbituric acid or 6-amino-1,3-dimethyluracil. Various conditions for these reactions have been reported [21-31]. Although these procedures

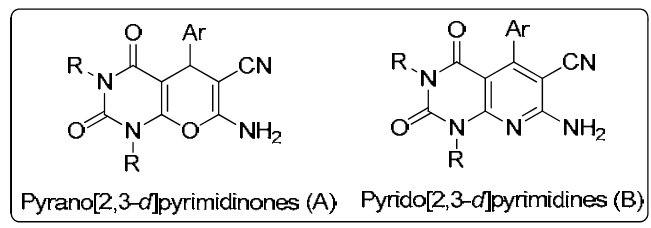

Fig. 1. The structure of pyrano[2,3-d]pyrimidinones (A) and pyri$\operatorname{do}[2,3-d]$ pyrimidines (B).

\footnotetext{
* Corresponding author. E-mail: shirini@guilan.ac.ir DOI: 10.1016/S1872-2067(17)62827-4| http://www.sciencedirect.com/science/journal/18722067 | Chin. J. Catal., Vol. 38, No. 7, July 2017
} 
are better than other methods, they have disadvantages such as long reaction time, harsh reaction conditions, the need for excess amounts of reagents, the use of organic solvents and toxic reagents, and non-recoverability of the catalyst. The development of simple, efficient, and mild procedures using easily separable and reusable solid catalysts to overcome these problems is therefore needed.

\section{Experimental}

\subsection{General}

Chemicals were purchased from Fluka, Merck, and Aldrich. The products were characterized by comparison of their melting points, and Fourier-transform infrared (FT-IR) and nuclear magnetic resonance (NMR) spectra with those of authentic samples and those reported in the literature. All yields refer to isolated products. Thin-layer chromatography (TLC; Polygram SILG/UV 254 plates) was used to determine the substrate purity and monitor the reaction. FT-IR spectra were recorded with a VERTEX 70 (Brucker, Germany) instrument using $\mathrm{KBr}$ disks. ${ }^{1} \mathrm{H}$ NMR and ${ }^{13} \mathrm{C}$ NMR spectra were recorded using a 400 $\mathrm{MHz}$ Bruker Avance instrument, with tetramethylsilane as an internal standard. Melting points were determined using an Electrothermal 9100 instrument and are uncorrected. Mass spectrometry (MS) was performed using an Agilent Technology (HP) instrument at $70 \mathrm{eV}$.

\subsection{General procedure for synthesis of 1,1'-(butane-1,4-diyl)bis} (1,4-diazabicyclo[2.2.2]octan-1-ium) chloride $\left(\left[C_{4}(D A B C O)_{2}\right] \cdot 2 C l\right)$

1,4-Dichlorobutane $(0.547 \mathrm{~mL}, 5.0 \mathrm{mmol})$ was added to DABCO (1.121 g, $10.0 \mathrm{mmol}$ ) and the mixture was stirred for 4 $\mathrm{h}$ at $80{ }^{\circ} \mathrm{C}$. After completion of the reaction, the solid product was washed with diethyl ether and dried under vacuum. $\left[\mathrm{C}_{4}(\mathrm{DABCO})_{2}\right] \cdot 2 \mathrm{Cl}$ was obtained as a white solid in $98.7 \%$ yield (1.39 g).

\subsection{General procedure for synthesis of 1,1'-(butane-1,4-diyl)bis (1,4-diazabicyclo[2.2.2]octan-1-ium) hydroxide $\left(\left[C_{4}(\mathrm{DABCO})_{2}\right] \cdot 2 \mathrm{OH}\right)$}

The amount of chloride in $\left[\mathrm{C}_{4}(\mathrm{DABCO})_{2}\right] \cdot 2 \mathrm{Cl}$ was determined by potentiometric titration to calculate the amount of $\mathrm{KOH}$ needed for complete ion exchange. For this purpose, 10 $\mathrm{mL}$ of $\left[\mathrm{C}_{4}(\mathrm{DABCO})_{2}\right] \cdot 2 \mathrm{Cl}$ in water $(0.05 \mathrm{~mol} / \mathrm{L})$ was titrated with $\mathrm{AgNO}_{3}(0.55 \mathrm{~mol} / \mathrm{L})$; the end-point occurred after consumption of $20 \mathrm{~mL}$ of $\mathrm{AgNO}_{3}$. This indicates that [ $\left.\mathrm{C}_{4}(\mathrm{DABCO})_{2}\right] \cdot 2 \mathrm{Cl}$ contains 2 equiv of $\mathrm{Cl}^{-}$per mole.

In the next step, solid $\mathrm{KOH}(0.561 \mathrm{~g}, 10 \mathrm{mmol})$ was added to a solution of [ $\left.\mathrm{C}_{4}(\mathrm{DABCO})_{2}\right] \cdot 2 \mathrm{Cl}(1.44 \mathrm{~g}, 5 \mathrm{mmol})$ in dry methanol and the mixture was stirred at room temperature (r.t.) for 3 h. The produced solid $(\mathrm{KCl})$ was removed by filtration, and the filtrate was evaporated under reduced pressure. The obtained viscous liquid was washed with ethyl acetate $(3 \times 10 \mathrm{~mL})$ and dried to give the pure IL, i.e., $\left[\mathrm{C}_{4}(\mathrm{DABCO})_{2}\right] \cdot 2 \mathrm{OH}(1.35 \mathrm{~g}$, $96.42 \%$ ) (Scheme 1).

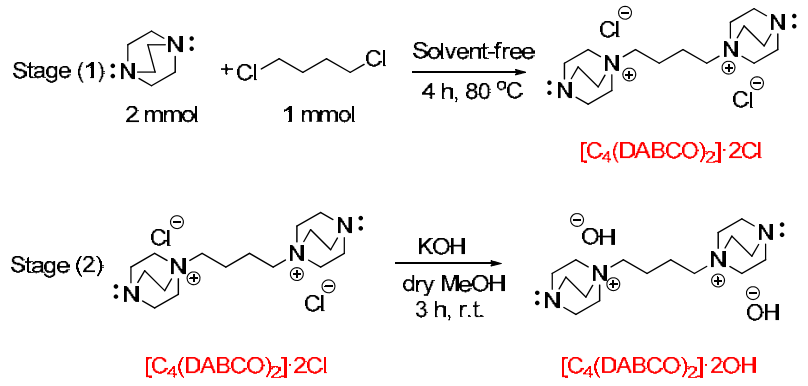

Scheme 1. Synthesis of $\left[\mathrm{C}_{4}(\mathrm{DABCO})_{2}\right] \cdot 2 \mathrm{OH}$.

\subsection{Catalyst characterization}

\subsubsection{FT-IR analysis}

The FT-IR spectra of DABCO and $\left[\mathrm{C}_{4}(\mathrm{DABCO})_{2}\right] \cdot 2 \mathrm{OH}$ are shown in Fig. 2. The DABCO spectrum shows bands from various stretching and bending vibrations. These bands are of lower intensity in the spectrum of $\left[\mathrm{C}_{4}(\mathrm{DABCO})_{2}\right] \cdot 2 \mathrm{OH}$, or are absent. The strong and broad band between 2700 and $3750 \mathrm{~cm}^{-1}$ (centered at $3424 \mathrm{~cm}^{-1}$ ) in the spectrum of [ $\left.\mathrm{C}_{4}(\mathrm{DABCO})_{2}\right] \cdot 2 \mathrm{OH}$ can be attributed to the hydroxide stretching mode. The strong peak at $1407 \mathrm{~cm}^{-1}$ and the small shoulder peak at $702 \mathrm{~cm}^{-1}$ are assigned to the scissoring and rocking vibrational modes, respectively, of the $\mathrm{CH}_{2}$ group.

\subsection{2. ${ }^{1} H$ NMR analysis}

The ${ }^{1} \mathrm{HNMR}$ spectrum of $\left[\mathrm{C}_{4}(\mathrm{DABCO})_{2}\right] \cdot 2 \mathrm{OH}$ is show in Fig. 3. The broad multiple peak at $1.7 \mathrm{ppm}$ can be attributed to chain hydrogen atoms $\left(\mathrm{H}_{\mathrm{a}}\right)$. The triplet peaks at 3.03 and 3.33 ppm are related to the cyclic hydrogens $\mathrm{H}_{\mathrm{d}}$ and $\mathrm{H}_{\mathrm{c}}$, respectively. The peak at $3.37 \mathrm{ppm}$, which can be assigned to $\mathrm{H}_{c}$ cyclic hydrogens, confirms that $\left[\mathrm{C}_{4}(\mathrm{DABCO})_{2}\right] \cdot 2 \mathrm{OH}$ was synthesized.

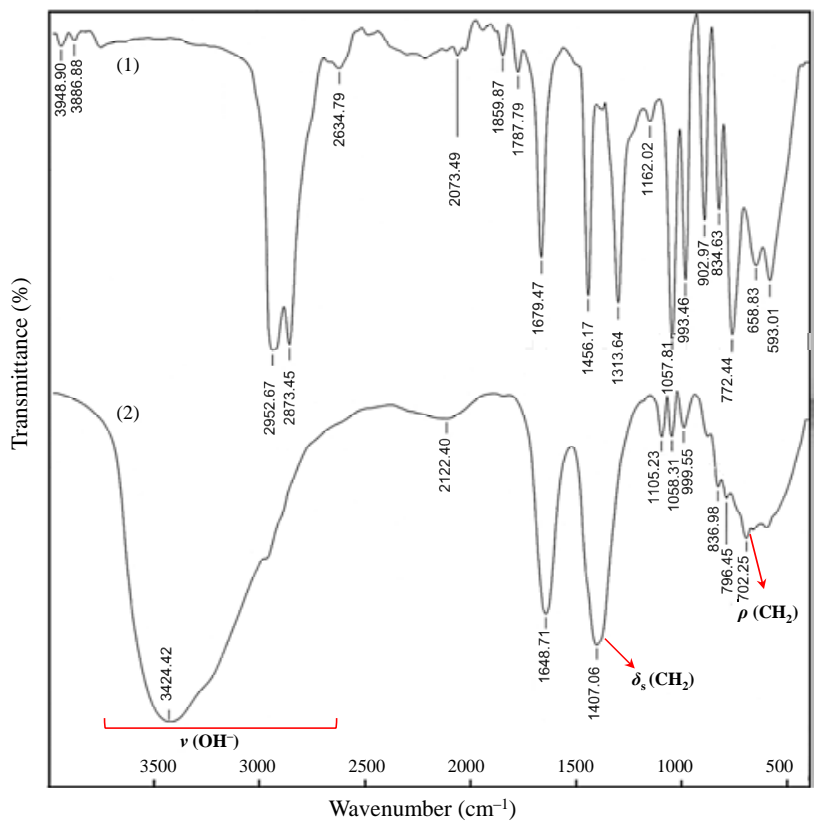

Fig. 2. FT-IR spectra of DABCO (1) and $\left[\mathrm{C}_{4}(\mathrm{DABCO})_{2}\right] \cdot 2 \mathrm{OH}(2)$. 


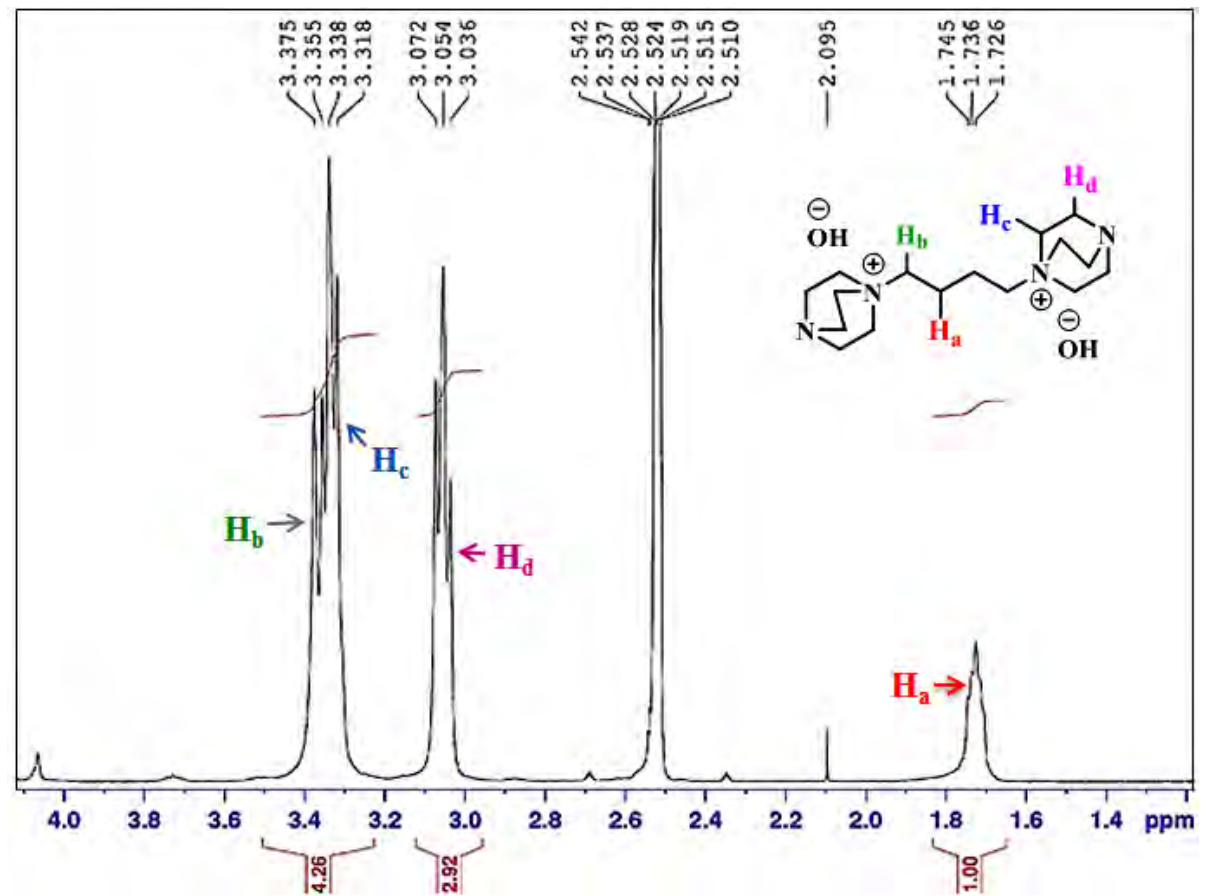

Fig. 3. ${ }^{1} \mathrm{H}$ NMR spectrum of $\left[\mathrm{C}_{4}(\mathrm{DABCO})_{2}\right] \cdot 2 \mathrm{OH}$.

\subsubsection{Titration curve for $\left[\mathrm{C}_{4}(\mathrm{DABCO})_{2}\right] \cdot 2 \mathrm{OH}$ with $\mathrm{HCl}$}

The basicity of $\left[\mathrm{C}_{4}(\mathrm{DABCO})_{2}\right] \cdot 2 \mathrm{OH}$ was determined by acid-base titration. An aqueous solution of the IL $(0.05 \mathrm{~mol} / \mathrm{L}, 10$ $\mathrm{mL})$ was titrated with a standard solution of $\mathrm{HCl}(0.1 \mathrm{~mol} / \mathrm{L})$ using a calibrated glass electrode $\mathrm{pH}$ meter at $25{ }^{\circ} \mathrm{C}$. Fig. 4 shows that $20 \mathrm{~mL}$ of $\mathrm{HCl}$ were needed to neutralize all the basic groups in $\left[\mathrm{C}_{4}(\mathrm{DABCO})_{2}\right] \cdot 2 \mathrm{OH}$. According to equation (1), $5 \mathrm{~mL}$ of $\mathrm{HCl}$ are needed to neutralize each basic group, therefore it can be concluded that this IL has four basic groups. Neutralization occurred in two stages, which indicates that $\left[\mathrm{C}_{4}(\mathrm{DABCO})_{2}\right] \cdot 2 \mathrm{OH}$ has two types of basic group. These results confirm the structure of the prepared reagent.

$$
\begin{gathered}
M_{(\text {acid) }} \times V_{\text {(acid) }}=M_{(\text {base })} \times V_{(\text {base })} \\
0.05(\mathrm{~mol} / \mathrm{L}) \times 10(\mathrm{~mL})=0.10(\mathrm{~mol} / \mathrm{L}) \times V_{(\text {acid })} \\
V_{\text {(acid) }}=5 \mathrm{~mL}
\end{gathered}
$$

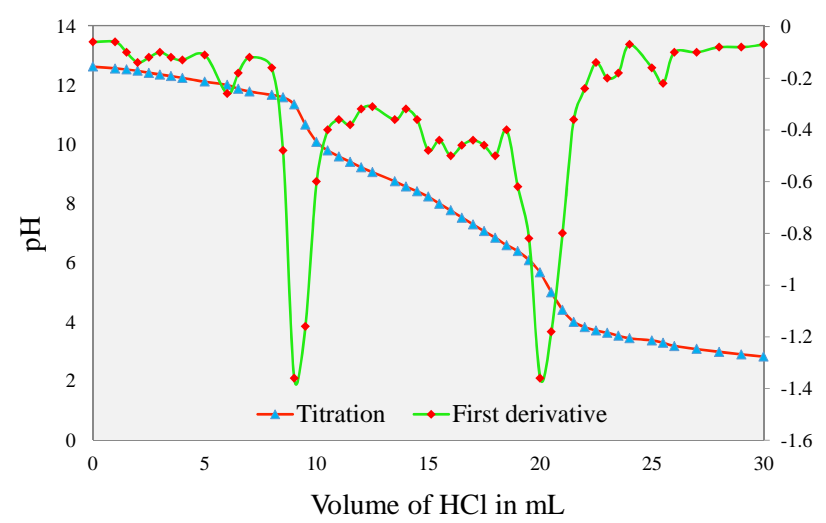

Fig. 4. Titration curve and its first derivative for IL with $\mathrm{HCl}(0.1$ $\mathrm{mol} / \mathrm{L})$.

\subsubsection{Leaching test}

The probability of hydroxide groups leaching from the $\left[\mathrm{C}_{4}(\mathrm{DABCO})_{2}\right] \cdot 2 \mathrm{OH}$ framework was determined using an in situ filtration technique. [ $\left.\mathrm{C}_{4}(\mathrm{DABCO})_{2}\right] \cdot 2 \mathrm{OH}(50 \mathrm{mg})$ was stirred in dichloromethane $(10 \mathrm{~mL})$ for $24 \mathrm{~h}$ at room temperature. The $\left[\mathrm{C}_{4}(\mathrm{DABCO})_{2}\right] \cdot 2 \mathrm{OH}$ was separated by filtration, and the filtrate was transferred to a $50 \mathrm{~mL}$ round-bottomed flask and evaporated to dryness. After drying, the $\mathrm{pH}$ values of fresh and tested $\left[\mathrm{C}_{4}(\mathrm{DABCO})_{2}\right] \cdot 2 \mathrm{OH}$ were determined using $\mathrm{pH}$-indicator strips. The images of the $\mathrm{pH}$-indicator strips show that no leaching of the basic hydroxyl groups occurred (Fig. 5).

\subsection{General procedure for synthesis of 2-amino-3-cyano-4H- pyran derivatives}

A mixture of an aldehyde ( $1 \mathrm{mmol})$, malononitrile ( $1 \mathrm{mmol})$, barbituric acid or 6-amino-1,3-dimethyluracil (1 mmol), and $\left[\mathrm{C}_{4}(\mathrm{DABCO})_{2}\right] \cdot 2 \mathrm{OH}(2 \mathrm{~mol} \%)$ was prepared. The mixture was ground for 5-11 min at room temperature using a mortar and

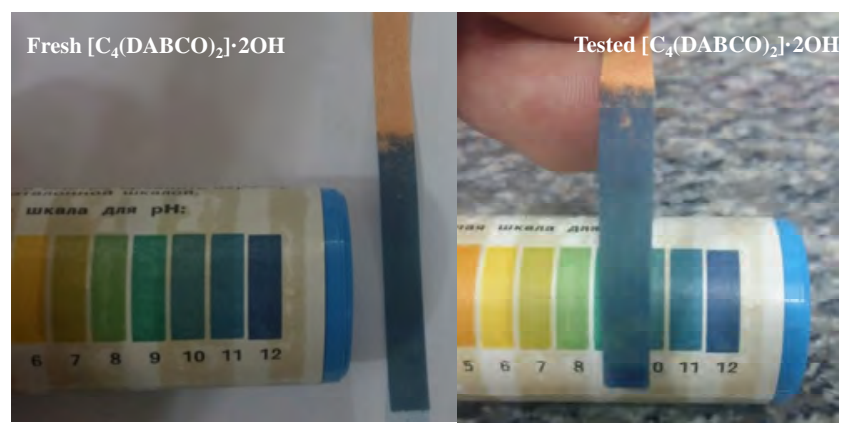

Fig. 5. Images of pH-indicator strips for fresh and tested $\left[\mathrm{C}_{4}(\mathrm{DABCO})_{2}\right] \cdot 2 \mathrm{OH}$. 

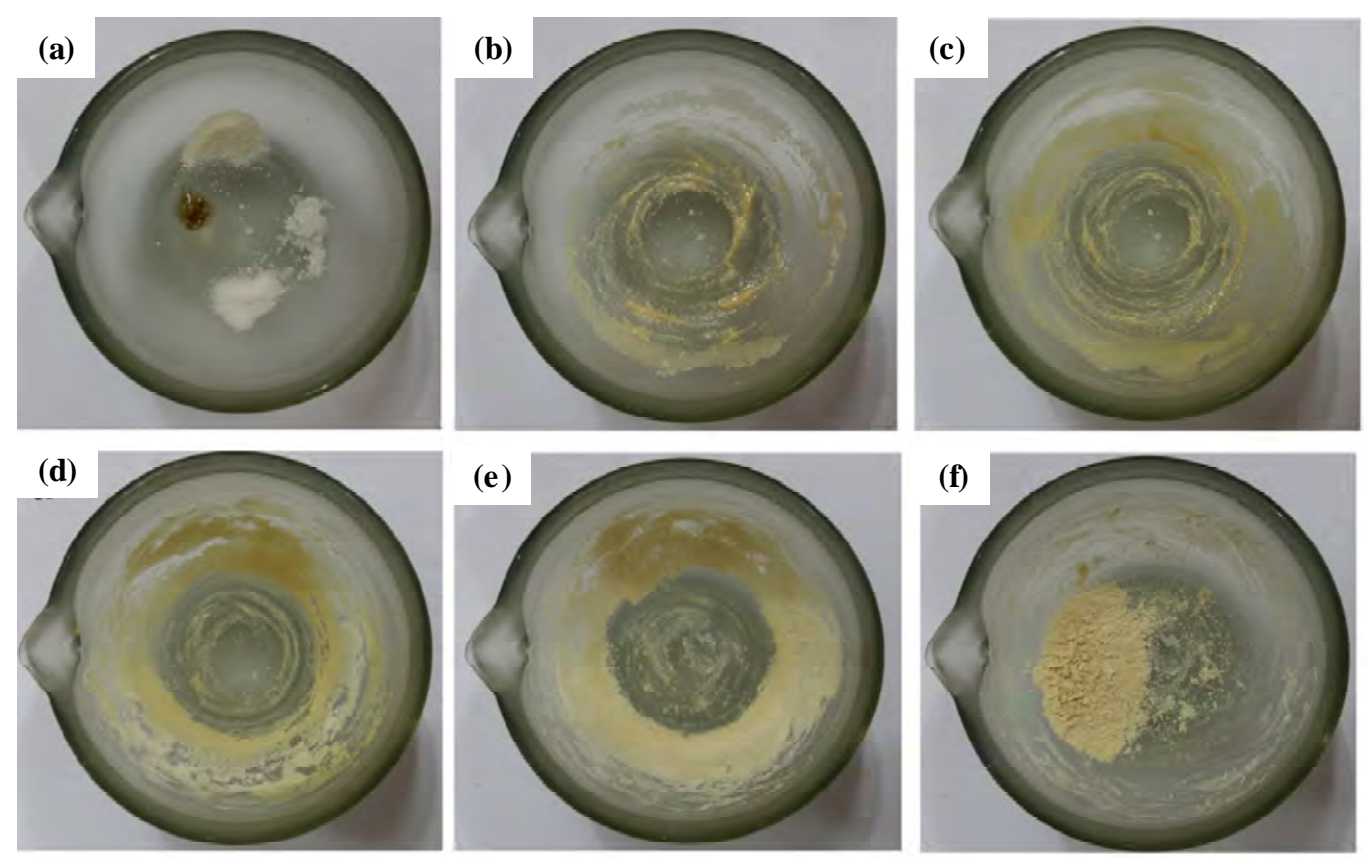

Fig. 6. Reaction process (a-f) using grinding method.

pestle; a solid mass was obtained (Fig. 6). After completion of the reaction (monitored using TLC), water ( $3 \mathrm{~mL}$ ) was added to the mortar, and the mixture was filtered to separate the crude product. The product was purified by recrystallization from ethanol.

The spectral data of new compounds are as follows.

7-Amino-2,4-dioxo-5-(o-tolyl)-1,3,4,5-tetrahydro-2H-pyrano [2,3- $d$ ] pyrimidine-6-carbonitrile (Table 1, entry 5): m.p. = 223-225 ${ }^{\circ}$ C; FT-IR (KBr, cm-1): 3302, 3070, 2962, 2223, 1698, $1606,1481,1377,1275,1212,1065$; MS: $m / z=297\left(\mathrm{M}^{+}+1\right)$;

\section{Table 1}

Preparation of pyrano[2,3- $d$ ]pyrimidinone derivatives using $\left[\mathrm{C}_{4}(\mathrm{DABCO})_{2}\right] \cdot 2 \mathrm{OH}$ as catalyst.

\begin{tabular}{|c|c|c|c|c|c|}
\hline \multirow{2}{*}{ Entry } & \multirow{2}{*}{ Aldehyde } & \multirow{2}{*}{$\begin{array}{l}\text { Time } \\
\text { (min) }\end{array}$} & \multirow{2}{*}{$\begin{array}{c}\text { Yield }^{a} \\
(\%)\end{array}$} & \multicolumn{2}{|c|}{ m.p. $\left({ }^{\circ} \mathrm{C}\right)$} \\
\hline & & & & Found & Reported \\
\hline 1 & $\mathrm{C}_{6} \mathrm{H}_{5} \mathrm{CHO}$ & 5 & 92 & $215-217$ & $215-217[30]$ \\
\hline 2 & $2-\mathrm{ClC}_{6} \mathrm{H}_{4} \mathrm{CHO}$ & 6 & 94 & $211-212$ & $211-212[30]$ \\
\hline 3 & $2-\mathrm{NO}_{2} \mathrm{C}_{6} \mathrm{H}_{4} \mathrm{CHO}$ & 4 & 95 & $253-256$ & $253-256[30]$ \\
\hline 4 & $2-\mathrm{OHC}_{6} \mathrm{H}_{4} \mathrm{CHO}$ & 7 & 95 & $160-162$ & $160-162[30]$ \\
\hline 5 & $2-\mathrm{CH}_{3} \mathrm{C}_{6} \mathrm{H}_{4} \mathrm{CHO}$ & 6 & 94 & $223-225$ & - \\
\hline 6 & $3-\mathrm{ClC}_{6} \mathrm{H}_{4} \mathrm{CHO}$ & 6 & 90 & $240-241$ & $240-241[30]$ \\
\hline 7 & $3-\mathrm{NO}_{2} \mathrm{C}_{6} \mathrm{H}_{4} \mathrm{CHO}$ & 5 & 95 & $267-269$ & $267-269[30]$ \\
\hline 8 & $4-\mathrm{ClC}_{6} \mathrm{H}_{4} \mathrm{CHO}$ & 5 & 94 & $245-247$ & $245-247[30]$ \\
\hline 9 & $4-\mathrm{BrC}_{6} \mathrm{H}_{4} \mathrm{CHO}$ & 5 & 94 & $231-233$ & $231-233$ [30] \\
\hline 10 & $4-\mathrm{OCH}_{3} \mathrm{C}_{6} \mathrm{H}_{4} \mathrm{CHO}$ & 4 & 96 & $280-281$ & $280-281[30]$ \\
\hline 11 & $4-\mathrm{NO}_{2} \mathrm{C}_{6} \mathrm{H}_{4} \mathrm{CHO}$ & 6 & 93 & $236-237$ & $237-238$ [30] \\
\hline 12 & $4-\mathrm{CH}_{3} \mathrm{C}_{6} \mathrm{H}_{4} \mathrm{CHO}$ & 6 & 85 & $225-227$ & $225[30]$ \\
\hline 13 & 4- $\mathrm{NMe}_{2} \mathrm{C}_{6} \mathrm{H}_{4} \mathrm{CHO}$ & 10 & 92 & $231-233$ & $230-232$ [30] \\
\hline 14 & Isatin & 4 & 95 & 243-245 & - \\
\hline 15 & $\begin{array}{c}\text { Pyridine-4-carbal- } \\
\text { dehyde }\end{array}$ & 7 & 94 & $211-212$ & - \\
\hline
\end{tabular}

a Isolated yields.
${ }^{1} \mathrm{H}$ NMR (DMSO- $d_{6}, 400 \mathrm{MHz}, \mathrm{ppm}$ ): $\delta=2.45$ (s, $3 \mathrm{H}, \mathrm{CH}_{3}$ ), 4.52 (s, 1H, CH) , 7.10-7.16 (m, 6H, ArH and $\left.\mathrm{NH}_{2}\right), 11.059$ (s, 1H, NH), $12.080(\mathrm{~s}, 1 \mathrm{H}, \mathrm{NH})$.

7'-Amino-2,2',4'-trioxo-1',2',3',4'-tetrahydrospiro[indoline3,5'-pyrano[2,3- $d$ ]pyrimidine]-6'-carbonitrile (Table 1, entry 14): m.p. $=243-245{ }^{\circ} \mathrm{C}$; FT-IR (neat, $\mathrm{cm}^{-1}$ ): 3352, 3303, 3140, $2202,1723,1672,1531,1389,1333,1108,995,635 ;$ MS: $m / z=$ $323\left(\mathrm{M}^{+}\right)$; ${ }^{1} \mathrm{H}$ NMR (DMSO- $\left.d_{6}, 400 \mathrm{MHz}, \mathrm{ppm}\right): \delta=6.8(\mathrm{~d}, J=7.6$ $\mathrm{Hz}, 1 \mathrm{H}), 6.9\left(1 \mathrm{H}, \mathrm{td}, J_{1}=7.6 \mathrm{~Hz}, J_{2}=0.8 \mathrm{~Hz}\right), 7.2(2 \mathrm{H}, \mathrm{m}), 7.4(2 \mathrm{H}$, s, $\left.\mathrm{NH}_{2}\right), 10.5(1 \mathrm{H}, \mathrm{s}, \mathrm{NH}), 11.145(1 \mathrm{H}, \mathrm{s}, \mathrm{NH}), 12.3(1 \mathrm{H}, \mathrm{s}, \mathrm{NH})$; ${ }^{13} \mathrm{C}$ NMR (DMSO- $d_{6}, 100 \mathrm{MHz}, \mathrm{ppm}$ ): $\delta=58,87,109,117,122$, $124,128,133,142,149,153,158,161,178$.

7-Amino-2,4-dioxo-5-(pyridin-4-yl)-1,3,4,5-tetrahydro- $2 \mathrm{H}$ pyrano[2,3- $d$ ] pyrimidine-6-carbonitrile (Table 1, entry 15): m.p. $=211-212^{\circ} \mathrm{C}$; FT-IR (neat, $\mathrm{cm}^{-1}$ ) 3418, 3316, 3179, 2958, 2883, 2187, 1683, 1599, 1369, 1214, 1146, 1040; MS: $m / z=$ $283\left(\mathrm{M}^{+}\right)$; ${ }^{1} \mathrm{H}$ NMR (DMSO- $\left.d_{6}, 500 \mathrm{MHz}, \mathrm{ppm}\right): \delta=4.38(\mathrm{a}, 1 \mathrm{H}$, $\mathrm{CH}), 7.28\left(2 \mathrm{H}, \mathrm{s}, \mathrm{NH}_{2}\right), 7.46-7.49(1 \mathrm{H}, \mathrm{dd}, \mathrm{ArH}), 7.82-7.85(1 \mathrm{H}$, dd, ArH), 8.5-8.52 (1H, dd, ArH), 8.55-8.58 (1H, dd, ArH), 9.38 $(1 \mathrm{H}, \mathrm{s}, \mathrm{NH}), 11.12(1 \mathrm{H}, \mathrm{s}, \mathrm{NH}) ;{ }^{13} \mathrm{C}$ NMR (DMSO- $d_{6}, 100 \mathrm{MHz}$, ppm): $\delta=26.02,33.92,57.83,82.83,87.6,114.80,115.26$, 119.48, 124.93, 138.06, 138.99, 141.03, 146.68, 146.83, 150.09, $153.16,158.31,163.05,164.58$.

7-Amino-5-(4-cyanophenyl)-1,3-dimethyl-2,4-dioxo-1,2,3,4tetrahydropyrido[2,3- $d$ ]pyrimidine-6-carbonitrile (Table 2, entry 9): m.p. > $300{ }^{\circ} \mathrm{C}$; FT-IR (KBr, cm-1): 3469, 3319, 3217, 2212, 1708, 1649, 1622, 1554, 1508, 1276, 1226, 973, 850, 806, 752; MS: $m / z=332\left(\mathrm{M}^{+}+1\right)$; ${ }^{1} \mathrm{H}$ NMR (DMSO- $d_{6}, 500 \mathrm{MHz}$, ppm): $\delta=3.09\left(\mathrm{~s}, 3 \mathrm{H}, \mathrm{CH}_{3}\right), 3.53\left(\mathrm{~s}, 3 \mathrm{H}, \mathrm{CH}_{3}\right), 7.28-7.31(\mathrm{~d}, 2 \mathrm{H})$, 7.36 (s, $2 \mathrm{H}, \mathrm{NH}_{2}$ ), 7.44-7.45 (d, 2H); ${ }^{13} \mathrm{C}$ NMR (DMSO- $d_{6}, 100$ $\mathrm{MHz}, \mathrm{ppm}): \delta=24.56,28.21,28.31,30.12,34.85,35.43,43.05$, 88.96, 99.11, 114.64, 115.76, 127.64, 128.01, 128.19, 128.73, 129.20, 134.99, 137.22, 151.36, 154.10, 158.91, 159.39, 160.75, 166.81 . 
Table 2

Preparation of pyrido[2,3- $d$ ]pyrimidine derivatives using $\left[\mathrm{C}_{4}(\mathrm{DABCO})_{2}\right] \cdot 2 \mathrm{OH}$ as catalyst.

\begin{tabular}{|c|c|c|c|c|c|}
\hline \multirow{2}{*}{ Entry } & \multirow{2}{*}{ Aldehyde } & \multirow{2}{*}{$\begin{array}{l}\text { Time } \\
\text { (min) }\end{array}$} & \multirow{2}{*}{$\begin{array}{c}\text { Yield }^{a} \\
(\%)\end{array}$} & \multicolumn{2}{|c|}{ m.p. $\left({ }^{\circ} \mathrm{C}\right)$} \\
\hline & & & & Found & Reported \\
\hline 1 & $\mathrm{C}_{6} \mathrm{H}_{5} \mathrm{CHO}$ & 8 & 92 & $>300$ & $>300[31]$ \\
\hline 2 & $2-\mathrm{ClC}_{6} \mathrm{H}_{4} \mathrm{CHO}$ & 11 & 93 & $>300$ & $>300[31]$ \\
\hline 3 & $3-\mathrm{ClC}_{6} \mathrm{H}_{4} \mathrm{CHO}$ & 8 & 92 & $>300$ & $>300[31]$ \\
\hline 4 & $4-\mathrm{ClC}_{6} \mathrm{H}_{4} \mathrm{CHO}$ & 7 & 94 & $>300$ & $>300[31]$ \\
\hline 5 & $3-\mathrm{NO}_{2} \mathrm{C}_{6} \mathrm{H}_{4} \mathrm{CHO}$ & 7 & 93 & $>300$ & $>300[31]$ \\
\hline 6 & $4-\mathrm{NO}_{2} \mathrm{C}_{6} \mathrm{H}_{4} \mathrm{CHO}$ & 8 & 95 & $>300$ & $>300[31]$ \\
\hline 7 & $3-\mathrm{BrC}_{6} \mathrm{H}_{4} \mathrm{CHO}$ & 8 & 92 & $>300$ & $>300[31]$ \\
\hline 8 & $4-\mathrm{FC}_{6} \mathrm{H}_{4} \mathrm{CHO}$ & 10 & 91 & $>300$ & $>300[31]$ \\
\hline 9 & 4- $\mathrm{CNC}_{6} \mathrm{H}_{4} \mathrm{CHO}$ & 10 & 93 & $>300$ & - \\
\hline 10 & 2-Naphthaldehyde & 8 & 91 & $>300$ & 一 \\
\hline
\end{tabular}

a Isolated yields.

7-Amino-1,3-dimethyl-5-(naphthalen-2-yl)-2,4-dioxo-1,2,3, 4-tetrahydropyrido[2,3- $d$ ]pyrimidine-6-carbonitrile (Table 2, entry 10): m.p. $>300{ }^{\circ} \mathrm{C}$; FT-IR (KBr, cm$\left.{ }^{-1}\right)$ : 3431, 3047, 3229, 2908, 2202, 1658, 1621, 1575, 1372, 1264, 741; MS: $m / z=357$ $\left(\mathrm{M}^{+}\right)$; ${ }^{1} \mathrm{H}$ NMR (DMSO- $\left.d_{6}, 500 \mathrm{MHz}, \mathrm{ppm}\right): \delta=3.07$ (s, $3 \mathrm{H}, \mathrm{CH}_{3}$ ), 3.55 (s, 3H, $\left.\mathrm{CH}_{3}\right), 7.35-7.37$ (dd, 2H), 7.57-7.63 (m, 2H), 7.81 (s, 1H), 7.92-8.01 (m, 3H); ${ }^{13} \mathrm{C}$ NMR (DMSO- $\left.d_{6}, 100 \mathrm{MHz}, \mathrm{ppm}\right): \delta=$ 19.05, 28.16, 30.14, 56.51, 89.11, 99.30, 115.95, 126.29, 126.33, $126.78,126.98,127.57,128.11,128.54,132.90,133.10,135.53$, $151.39,154.16,159.01,159.70,160.79$.

\section{Results and discussion}

The structure of $\left[\mathrm{C}_{4}(\mathrm{DABCO})_{2}\right] \cdot 2 \mathrm{OH}$ suggests that this reagent has good potential as a basic catalyst for reactions that are accelerated by this type of catalyst. We therefore investigated the use of this reagent for promotion of the synthesis of pyrano[2,3- $d]$ pyrimidinone and pyrido[2,3- $d]$ pyrimidine derivatives.

First, we optimized the reaction conditions in the synthesis of pyrano[2,3- $d$ ]pyrimidinone derivatives by performing the condensation of 4-chlorobenzaldehyde, barbituric acid, and malononitrile in the absence or presence of $\left[\mathrm{C}_{4}(\mathrm{DABCO})_{2}\right] \cdot 2 \mathrm{OH}$ under various conditions (Table 3 ). The best results were obtained using a grinding method with $10 \mathrm{~mol} \%$ of $\left[\mathrm{C}_{4}(\mathrm{DABCO})_{2}\right] \cdot 2 \mathrm{OH}$ at room temperature under solvent-free conditions.

After optimization of the reaction conditions, the effectiveness and suitability of this method were investigated by performing the reaction using a variety of simple and readily available substrates under the optimal conditions.

For this purpose, various aromatic aldehydes containing electron-withdrawing and electron-donating groups, i.e., $\mathrm{Cl}, \mathrm{Br}$, $\mathrm{CH}_{3}, \mathrm{OCH}_{3}$, and $\mathrm{NO}_{2}$, in the ortho, meta, and para positions of the benzene ring were reacted with barbituric acid and malononitrile under the optimal conditions. The corresponding
Table 3

Optimization of conditions for reactions catalyzed by $\left[\mathrm{C}_{4}(\mathrm{DABCO})_{2}\right] \cdot 2 \mathrm{OH}^{\mathrm{a}}$.

\begin{tabular}{lccccc}
\hline Entry & $\begin{array}{c}\text { Catalyst } \\
(\mathrm{mol} \%)\end{array}$ & Solvent & $\begin{array}{c}\text { Temperature } \\
\left({ }^{\circ} \mathrm{C}\right)\end{array}$ & $\begin{array}{c}\text { Time } \\
(\mathrm{min})\end{array}$ & $\begin{array}{c}\text { Yield }^{\mathrm{b}} \\
(\%)\end{array}$ \\
\hline 1 & - & $\mathrm{EtOH}$ & r.t. & 60 & Trace \\
2 & - & $\mathrm{H}_{2} \mathrm{O}$ & r.t. & 60 & Trace \\
3 & - & Grinding & r.t. & 60 & Trace \\
4 & 10 & EtOH & r.t. & 60 & 70 \\
5 & 10 & $\mathrm{H}_{2} \mathrm{O}$ & r.t. & 60 & 80 \\
7 & 10 & Grinding & r.t. & 5 & 94 \\
8 & 15 & Grinding & r.t. & 5 & 95 \\
9 & 5 & Grinding & r.t. & 25 & 85 \\
\hline
\end{tabular}

a Reaction conditions: 4-chlorobenzaldehyde (1 mmol), barbituric acid $(1 \mathrm{mmol})$, malononitrile $(1 \mathrm{mmol})$, in the presence of $\left[\mathrm{C}_{4}(\mathrm{DABCO})_{2}\right] \cdot 2 \mathrm{OH}$ as the catalyst.

b Isolated yields.

products were obtained in high isolated yields in short reaction times (Table 1, entries 1-13). The heterocyclic aldehydes isatin and pyridine-4-carbaldehyde were also used under these conditions, and the desired products were obtained in high yields (Table 1, entries 14 and 15).

Next, $\left[\mathrm{C}_{4}(\mathrm{DABCO})_{2}\right] \cdot 2 \mathrm{OH}$ was used to promote the condensation of aldehydes, malononitrile, and 6-amino-1,3-dimethyluracil to give pyrido[2,3- $d$ ]pyrimidines. The reaction conditions were optimized using the reaction between 4-chlorobenzaldehyde, malononitrile, and 6-amino1,3-dimethyluracil as a model reaction. The best results were obtained by grinding $1 \mathrm{mmol}$ of aldehyde, $1 \mathrm{mmol}$ of malononitrile, $1 \mathrm{mmol}$ of 6-amino-1,3-dimethyluracil, and $10 \mathrm{~mol} \%$ $\left[\mathrm{C}_{4}(\mathrm{DABCO})_{2}\right] \cdot 2 \mathrm{OH}$ at room temperature. Under the optimal conditions, various aromatic aldehydes containing electron-releasing and electron-withdrawing substituents on the aromatic ring gave the corresponding products in high yields in short reaction time (Table 2).

We compared the results obtained using our newly developed method for the $\left[\mathrm{C}_{4}(\mathrm{DABCO})_{2}\right] \cdot 2 \mathrm{OH}$-catalyzed synthesis of 7-amino-5-(4-chlorophenyl)-2,4-dioxo-1,3,4,5-tetrahydro-2H-p yrano[2,3- $d$ ]pyrimidine-6-carbonitrile (Table 1, entry 8) and 7-amino-5-(4-nitrophenyl)-2,4-dioxo-1,3,4,5-tetrahydro-2 $H$-py rano[2,3-d]pyrimidine-6-carbonitrile (Table 1, entry 11) with the results reported in the literature for other synthetic methods (Table 4). The data in Table 4 show that this method avoids some of the difficulties associated with other procedures such as long reaction time, low yields, harsh conditions for catalyst preparation, e.g., IR or microwave irradiation, and high catalyst loadings.

A proposed mechanism for the synthesis of pyrano[2,3- $d$ ] pyrimidinone and pyrido[2,3- $d$ ] pyrimidine derivatives is shown in Scheme 2. In this procedure, $\left[\mathrm{C}_{4}(\mathrm{DABCO})_{2}\right] \cdot 2 \mathrm{OH}$ effectively catalyzes the formation of olefin (V), which is readily prepared by Knoevenagel condensation of an aldehyde and active methylene nitrile (I), via intermediate (IV). Barbituric acid or 6-amino-1,3-dimethyluracil then reacts with V to give VII or VII', followed by intermolecular cyclization to VIII or VIII'. Finally, the products are formed by tautomerization. 
Table 4

Comparison of results obtained for synthesis of selected compounds in presence of $\left[\mathrm{C}_{4}(\mathrm{DABCO})_{2}\right] \cdot 2 \mathrm{OH}$ with those obtained using other catalysts.

\begin{tabular}{|c|c|c|c|c|c|}
\hline Product & Catalyst (mol\%) & Reaction condition & Time (min) & Yield $^{\mathrm{a}}(\%)$ & Ref. \\
\hline & $\mathrm{Zn}[(\mathrm{L}) \text { proline }]_{2}(17)$ & EtOH / reflux & 50 & 90 & {$[22]$} \\
\hline & {$\left[\mathrm{BMIm} \mathrm{BF}_{4}(1.5 \mathrm{~g})\right.$} & $90^{\circ} \mathrm{C}$ & 180 & 92 & {$[23]$} \\
\hline & DABCO`$^{`}(10)$ & Ethanol- $\mathrm{H}_{2} \mathrm{O}(1: 1)$ / r.t. & 120 & 89 & {$[25]$} \\
\hline & SBA-Pr-SO ${ }_{3} \mathrm{H}(0.02 \mathrm{~g})$ & Neat $/ 140^{\circ} \mathrm{C}$ & 45 & 30 & {$[26]$} \\
\hline & $\operatorname{KBr}(100)$ & Electrolysis & 20 & 77 & [29] \\
\hline & {$\left[\mathrm{C}_{4}(\mathrm{DABCO})_{2}\right] \cdot 2 \mathrm{OH}(10)$} & Grinding/ r.t. & 5 & 94 & This work \\
\hline & L-proline (5) & aq. EtOH, r.t. & 45 & 73 & {$[21]$} \\
\hline & $\mathrm{Zn}[(\mathrm{L}) \text { proline }]_{2}(17)$ & EtOH / reflux & 30 & 92 & {$[22]$} \\
\hline & DAHP (10) & aq. EtOH / r.t. & 120 & 72 & {$[24]$} \\
\hline & $\mathrm{DABCO}^{`}(10)$ & Ethanol- $\mathrm{H}_{2} \mathrm{O}(1: 1)$ / r.t & 120 & 92 & {$[25]$} \\
\hline & SBA-Pr-SO ${ }_{3} \mathrm{H}(0.02 \mathrm{~g})$ & Neat $/ 140^{\circ} \mathrm{C}$ & 15 & 90 & [26] \\
\hline & ТВАВ (10) & $\mathrm{H}_{2} \mathrm{O} /$ reflux & 35 & 80 & [27] \\
\hline $\mathrm{H}$ & $\mathrm{KBr}(100)$ & Electrolysis & 20 & 73 & [29] \\
\hline & {$\left[\mathrm{C}_{4}(\mathrm{DABCO})_{2}\right] \cdot 2 \mathrm{OH}(10)$} & Grinding/ r.t. & 6 & 93 & This work \\
\hline
\end{tabular}

a Isolated yields.

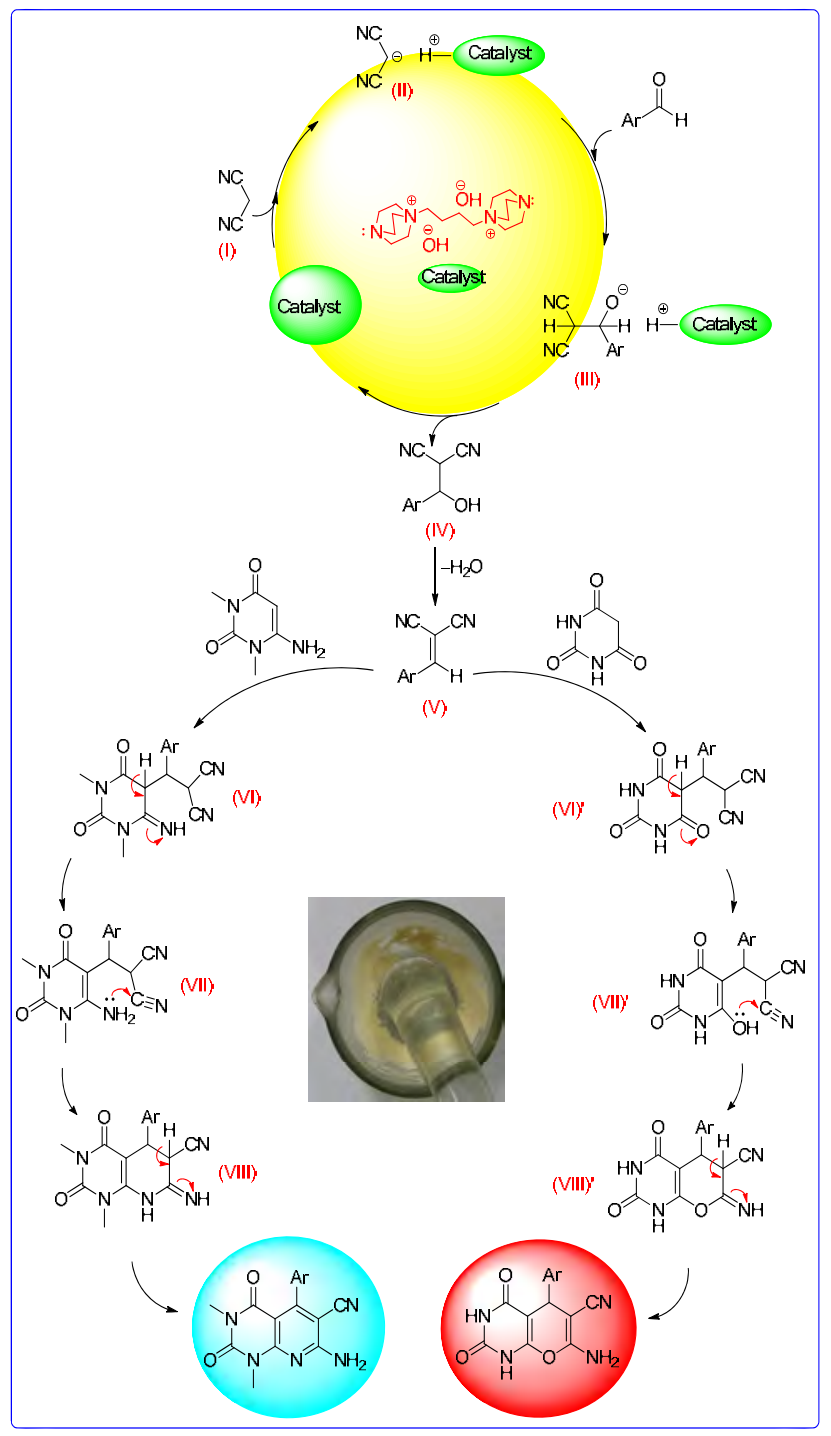

Scheme 2. Proposed mechanism for synthesis of pyrano[2,3- $d]$ pyrimidinone and pyrido[2,3- $d]$ pyrimidine derivatives using $\left[\mathrm{C}_{4}(\mathrm{DABCO})_{2}\right] \cdot 2 \mathrm{OH}$ as catalyst.

\section{Conclusions}

In summary, we synthesized pyrano[2,3- $d$ ]pyrimidinone and pyrido[2,3- $d]$ pyrimidine derivatives using 1,1'-(butane1,4-diyl)bis(1,4-diazabicyclo[2.2.2]octan-1-ium) hydroxide as an effective IL catalyst with dual basic functional groups. This synthesis has various advantages: preparation of the basic IL catalyst is simple, and the IL is not harmful to the environment; the starting materials are readily available; mild reaction conditions can be used; the reaction profile is clean; high reaction rates and excellent yields are achieved; and column chromatographic separation of the products is not needed.

\section{Acknowledgments}

The authors are thankful to the University of Guilan Research Council for the partial support of this work.

\section{References}

[1] A. Mulik, D. Chandam, P. Patil, D. Patil, S. Jagdale, M. Deshmukh, J. Mol. Liq., 2013, 179, 104-109.

[2] J. M. Xu, B. K. Liu, W. B. Wu, C. Qian, Q. Wu, X. F. Lin, J. Org. Chem., 2006, 71, 3991-3993.

[3] A. R. Hajipour, F. Rafiee, J. Iran. Chem. Soc., 2009, 6, 647-678.

[4] K. Niknam, N. Borazjani, R. Rashidian, A. Jamali, Chin. J. Catal., 2013, 34, 2245-2254.

[5] S. I. Alqasoumi, A. M. Al-Taweel, A. M. Alafeefy, E. Noaman, M. M. Ghorab, Eur. J. Med. Chem., 2010, 45, 738-744.

[6] A. D. Broom, J. L. Shim, G. L. Anderson, J. Org. Chem., 1976, 41, 1095-1099.

[7] A. A. Joshi, C. L. Viswanathan, Anti-Infect. Agents Med. Chem., 2006, $5,105-122$.

[8] M. M. Ghorab, A. Y. Hassan, Phosphorus, Sulfur Silicon Relat. Elem., 1998, 141, 251-261.

[9] P. Sharma, N. Rane, V. K. Gurram, Bioorg. Med. Chem. Lett., 2004, $14,4185-4190$.

[10] A. B. A. El-Gazzar, H. N. Hafez, G. A. M. Nawwar, Eur. J. Med. Chem., 2009, 44, 1427-1436. 


\title{
Graphical Abstract
}

Chin. J. Catal., 2017, 38: 1245-1251 doi: 10.1016/S1872-2067(17)62827-4

Efficient synthesis of pyrano[2,3-d]pyrimidinone and pyrido[2,3- $d]$ pyrimidine derivatives in presence of novel basic ionic liquid catalyst

Omid Goli Jolodar, Farhad Shirini *, Mohadeseh Seddighi University of Guilan, Iran

1,1'-(Butane-1,4-diyl)bis(1,4-diazabicyclo[2.2.2]octan-1-ium) hydroxide, which is a basic ionic liquid, efficiently promoted the synthesis of pyrano[2,3- $d]$ pyrimidinones and pyrido[2,3- $d]$ pyrimidines at room temperature under grinding conditions.

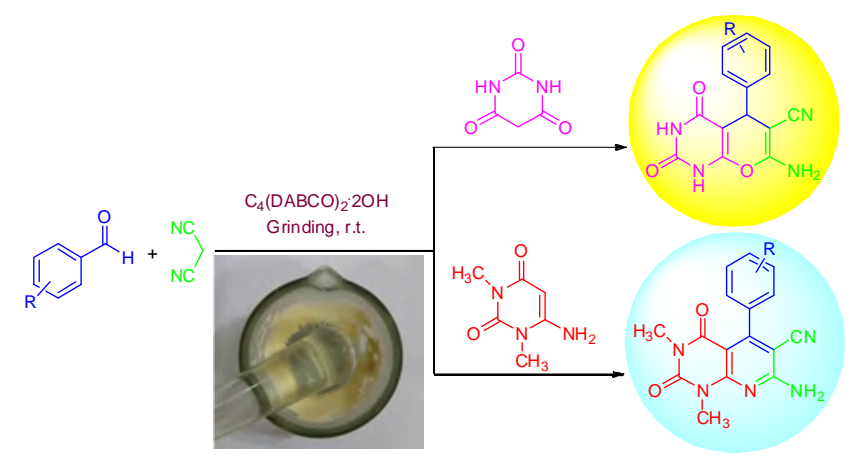

[11] R. Gupta, A. Jain, R. Joshi, M. Jain, Bull. Korean Chem. Soc., 2011, 32, 899-904.

[12] M. M. Hanna, Eur. J. Med. Chem., 2012, 55, 12-22.

[13] S. V. Shinde, W. N. Jadhav, N. N. Karade, Orient. J. Chem., 2010, 26, 307-317.

[14] R. B. Lichtner, G. Hutchinson, K. Hellmann, Eur. J. Cancer Clin. Oncol., 1989, 25, 945-951.

[15] J. I. DeGraw, P. H. Christie, W. T. Clowell, F. M. Sirotnak, J. Med. Chem., 1992, 35, 320-324.

[16] H. D. Thomas, K. Saravanan, L. Z. Wang, M. J. Lin, J. S. Northen, H. Barlow, M. Barton, D. R. Newell, R. J. Griffin, B. T. Golding, N. J. Curtin, Mol. Cancer Ther., 2009, 8, 1828-1837.

[17] J. P. De la Cruz, A. Moreno, F. Mérida, J. García-Campos, F. S. de la Cuesta, Pharmacol. Toxicol., 1994, 75, 250-254.

[18] N. C. Petal, A. G. Mehta, Asian J. Chem., 2001, 13, 1385-1388.

[19] B. S. Holla, M. Mahalinga, M. S. Karthikeyan, P. M. Akberali, N. S. Shetty, Bioorg. Med. Chem., 2006, 14, 2040-2047.

[20] S. Całus, E. Gondek, A. Danel, B. Jarosz, M. Pokładko, A. V. Kityk, Mater. Lett., 2007, 61, 3292-3295.

[21] M. Bararjanian, S. Balalaie, B. Movassagh, A. M. Amani, J. Iran.
Chem. Soc., 2009, 6, 436-442.

[22] M. M. Heravi, A. Ghods, K. Bakhtiari, F. Derikvand, Synth. Commun., 2010, 40, 1927-1931.

[23] J. Yu, H. Q. Wang, Synth. Commun., 2005, 35, 3133-3140.

[24] S. Balalaie, S. Abdolmohammadi, H. R. Bijanzadeh, A. M. Amani, Mol. Divers., 2008, 12, 85-91.

[25] J. Azizian, A. Shameli, S. Balalaie, M. M. Ghanbari, S. Zomorodbakhsh, M. Entezari, S. Bagheri, G. Fakhrpour, Orient. J. Chem., 2012, 28, 327-332.

[26] G. M. Ziarani, S. Faramarzi, S. Asadi, A. Badiei, R. Bazl, M. Amanlou, DARU J. Pharm. Sci., 2013, 21, 3.

[27] A. Mobinikhaledi, M. A. B. Fard, Acta. Chim. Slov., 2010, 57, 931-935.

[28] S. Mashkouri, M. R. Naimi-Jamal, Molecules, 2009, 14, 474-479.

[29] H. Kefayati, M. Valizadeh, A. Islamnezhad, Anal. Bioanal. Electrochem., 2014, 6, 80-90.

[30] O. Goli-Jolodar, F. Shirini, M. Seddighi, J. Iran. Chem. Soc., 2016, 13, 457-463.

[31] A. M. Rad, M. Mokhtary, Int. Nano Lett., 2015, 5, 109-123.

\section{新型碱性离子液体催化剂高效催化合成吡喃酮 [2,3-d]嘧啶酮和[2,3-d]嘧啶衍生物}

\author{
Omid Goli Jolodar, Farhad Shirini *, Mohadeseh Seddighi \\ 桂兰大学理学院化学系, 雷什特41335, 伊朗
}

\begin{abstract}
摘要: 制备了碱性离子液体1,1'-(丁烷-1,4-二基)双(1,4-二氮杂二环[2.2.2]辛烷-1-ium)羟化物, 并采用红外光谱、 ${ }^{1} \mathrm{H}$ 核磁共振 谱和 $\mathrm{pH}$ 值分析对其进行了表征. 然后将它用于室温研磨条件下高效催化合成吡喃酮[2,3- $d$ ] 密啶酮和[2,3- $d$ 嘧啶. 该法步骤 简单, 反应时间短, 产物收率高, 无需柱色谱分离, 原料易得, 且可回收利用.
\end{abstract}

关键词: 碱性催化剂; 多组分反应; 嘧啶衍生物; 研磨

收稿日期: 2017-01-21. 接受日期: 2017-03-15. 出版日期: 2017-07-05.

*通讯联系人. 电子信箱: shirini@guilan.ac.ir

本文的英文电子版由Elsevier出版社在ScienceDirect上出版(http://www.sciencedirect.com/science/journal/18722067). 